\title{
DNA methylation mediates differentiation in thermal responses of Pacific oyster (Crassostrea gigas) derived from different tidal levels
}

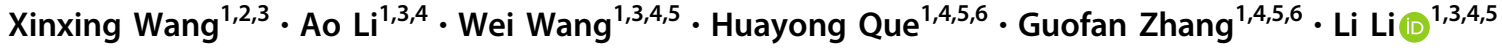

Received: 1 January 2020 / Revised: 6 July 2020 / Accepted: 28 July 2020 / Published online: 17 August 2020

(c) The Author(s), under exclusive licence to The Genetics Society 2020

\begin{abstract}
Epigenetic mechanisms such as DNA methylation have the potential to affect organism acclimatization and adaptation to environmental changes by influencing their phenotypic plasticity; however, little is known about the role of methylation in the adaptive phenotypic divergence of marine invertebrates. Therefore, in this study, a typical intertidal species, the Pacific oyster (Crassostrea gigas), was selected to investigate the epigenetic mechanism of phenotypic plasticity in marine invertebrates. Intertidal and subtidal oysters subjected to one-generation common garden experiments and exhibited phenotypic divergence were used. The methylation landscape of both groups of oysters was investigated under temperate and high temperature. The two tidal oysters exhibited divergent methylation patterns, regardless of the temperature, which was mainly original environment-induced. Intertidal samples exhibited significant hypomethylation and more plasticity of methylation in response to heat shock, while subtidal samples showed hypermethylation and less plasticity. Combined with RNA-seq data, a positive relationship between methylation and expression in gene bodies was detected on a genome-wide scale. In addition, approximately $11 \%$ and $7 \%$ of differentially expressed genes showed significant methylation variation under high temperatures in intertidal and subtidal samples, respectively. Genes related to apoptosis and organism development may be regulated by methylation in response to high temperature in intertidal oysters, whereas oxidationreduction and ion homeostasis-related genes were involved in subtidal oysters. The results also suggest that DNA methylation mediates phenotypic divergence in oysters adapting to different environments. This study provides new insight into the epigenetic mechanisms underlying phenotypic plasticity in adaptation to rapid climate change in marine organisms.
\end{abstract}

Supplementary information The online version of this article (https:// doi.org/10.1038/s41437-020-0351-7) contains supplementary material, which is available to authorized users.

$\mathrm{Li} \mathrm{Li}$

lili@qdio.ac.cn

1 CAS Key Laboratory of Experimental Marine Biology, Institute of Oceanology, Chinese Academy of Sciences, Qingdao 266071, China

2 University of Chinese Academy of Sciences, Beijing 100039, China

3 Laboratory for Marine Fisheries Science and Food Production Processes, Pilot National Laboratory for Marine Science and Technology, Qingdao 266237, China

4 National and Local Joint Engineering Laboratory of Ecological Mariculture, Qingdao 266071, China

5 Center for Ocean Mega-Science, Chinese Academy of Sciences, Qingdao 266071, China

6 Laboratory for Marine Biology and Biotechnology, Pilot National Laboratory for Marine Science and Technology, Qingdao 266237, China

\section{Introduction}

Phenotypic plasticity is a key adaptive mechanism for facing the challenges of changing natural conditions and human disturbances, as it allows a given genotype to produce different phenotypes (Holloway 2002; Williams et al. 2008). This is thought to be particularly important for species living in highly variable environments, because it serves as a buffer and enables the individual to match its phenotype to a range of stress conditions. The intertidal zone represents one of the most vulnerable and susceptible regions in the sea, as it shows dramatic variation on both spatial and temporal scales in abiotic and biotic conditions. Temperature is one of the major abiotic factors, and fluctuates widely in the intertidal region during daily and seasonal exposure to air (Southward 1958). These drastic changes in environmental temperature pose potentially critical challenges for ectothermic marine invertebrates, as changes in their body temperatures can directly modulate their rates of biochemical reactions and biological processes 
(Pörtner et al. 2007; Schulte et al. 2011; Somero 2010; Sunday et al. 2012).

One mechanism of phenotypic plasticity that can regulate gene expression and facilitate adaptation is epigenetics, which refers to processes capable of regulating gene activity without altering the underlying DNA sequence (Deans and Maggert 2015). Histone modifications, DNA methylation, and noncoding RNA activity (e.g., miRNA) are the most commonly described epigenetic modifications. Among these, DNA methylation is one of the best-characterized epigenetic mechanisms due to the ease of study and rapidly growing amount of literatures, whose capacity to shape fitness-related phenotypes has attracted the interest of ecologists and evolutionary biologists. The importance of DNA methylation in development differentiation has been highlighted by previous studies in fish, sea turtles, and oysters (Ellison et al. 2015; Riviere et al. 2013; Venegas et al. 2016). Environment-induced variation in DNA methylation has also been investigated in marine invertebrates. For example, environmentally susceptible corals (Putnam et al. 2016), reef-building coral (Liew et al. 2018), and invasive colonial ascidians (Hawes et al. 2018) can adjust their DNA methylation status to regulate their phenotypic plasticity and thus rapidly adapt to ocean acidification and high temperatures. Changes, such as salinity stress (Zhang et al. 2017a), air exposure (Zhang et al. 2017b), and diuron exposure (Rondon et al. 2017), can also induce global DNA methylation variation in oysters. Studies have demonstrated that temperature stress can induce significant global DNA methylation changes in ascidians, Antarctic polychaetes, and the three-spine stickleback (Hawes et al. 2018; Marsh and Pasqualone 2014; Metzger and Schulte 2017). Most of these studies focus on direct environmental effects, but heritable epigenetic modification in offspring generations deserves further investigation, because it could make it possible to predict environmental effects and adaptive potential in the face of environmental change in marine organisms.

Fine-scale differentiation in nearby habitats can be mediated by DNA methylation. For example, individuals of Laguncularia racemosa from salt marsh and riverside exhibited little genetic but abundant methylation differentiation, implying the important role of epigenetic variation in coping with different environments in natural populations (Lira-Medeiros et al. 2010). In marine organisms, differentiation of phenotypic traits in thermal tolerance (Diederich and Pechenik 2013), flexibility of physiology (Fernandez-Reiriz et al. 2016), and transcriptome profiles (Place et al. 2012) between intertidal and subtidal animals have been reported in previous studies. However, the underlying epigenetic mechanisms remain unexplored. The sessile oyster, as one of the dominant species in the intertidal and shallow subtidal region, provides an ideal model to study adaptive phenotypic divergence. In our previous study, differentiation has been identified between intertidal and subtidal oysters subjected to one-generation common garden experiments in multiple traits, including growth, survival, physiological, and transcriptomic responses at high temperature ( $\mathrm{Li}$ et al. 2018). We propose that DNA methylation may underlie the adaptive phenotypic plasticity above. In this study, we attempted to further investigate DNA methylation as an epigenetic control mechanism underlying adaptive phenotypic divergence in Crassostrea gigas. By performing whole-genome bisulfite sequencing (WGBS) combined with RNA-seq analysis, this study linked epigenetic variation to gene expression. This was intended to enable us not only to explore the epigenetic basis underlying the mechanisms of adaptive phenotypic divergence that may modulate the capacity of organisms to cope with environmental change, but also to investigate the heritable epigenetic effects thereby induced. This study aims to support the understanding of acclimatization, and hence improve culture mode design for aquaculture (Mondol et al. 2016).

\section{Materials and methods}

\section{Common garden experiments and heat-stress treatment}

The breeding and the heat-stress treatment of the oysters used in this study were performed in a previous study ( $\mathrm{Li}$ et al. 2018). Two wild oyster groups collected from the intertidal and subtidal zones at Laoting $\left(39^{\circ} 16^{\prime} \mathrm{N}\right.$, Tangshan, Hebei province, China) were translocated to Qingdao for 1 month of acclimatization before spawning. The breeding was carried out under the condition of seawater at $22.5-23.5^{\circ} \mathrm{C}$ temperature and $29 \pm 0.5 \%$ o salinity. For each group, eggs from 30 mature female oysters were mixed and then divided into 30 . Each was fertilized individually with each of the 30 mature male oysters. The zygotes from each male individual were incubated in a $70-\mathrm{L}$ plastic container. At the D-shaped stage, larvae from six containers were combined to form one group with three culture replicates (Fig. S1). The rearing of larvae and spat was performed using standard practices (Guo et al. 2012). Spats were put into cages and cultured in the sea at a density of $\sim 100$ individuals per layer when the shell height reached $3 \mathrm{~cm}$. Cages were changed four times each year to decrease the density as the size of the oysters increased. All culture replicates were well maintained in the sea. The 10-monthold $\mathrm{F}_{1}$-generation oysters were acclimated in the laboratory aquarium (temperature: $14.2-18.2^{\circ} \mathrm{C}$; salinity: $31.3 \pm$ $0.5 \%$ ) for 15 days with aerated, filtered seawater before the 
experiments. Commercial spirulina powder was added as a food source, and seawater was changed daily.

Oysters of both groups were randomly selected and exposed to acute heat shock $\left(35^{\circ} \mathrm{C}\right)$ for $6 \mathrm{~h}$. Seawater was not changed, and the oysters were not fed during this period. The gills sampled at 0 and $6 \mathrm{~h}(n=15$ each sample time in each group) were immediately frozen in liquid nitrogen, and then stored at $-80^{\circ} \mathrm{C}$ for DNA extraction. InC/SubC and $\mathrm{InH} / \mathrm{SubH}$ were used to represent control and heatshock treatment samples from the intertidal/subtidal area.

\section{Library construction and bisulfite sequencing}

Genomic DNA was isolated from gills using a Marine Animals DNA Kit (TIANGEN, Beijing, China) following the manufacturer's recommended protocol. DNA from 15 individuals in each sampling group was equally mixed to generate three biological replicates of five individuals. A total amount of $5.2 \mu \mathrm{g}$ of genomic DNA spiked with $26 \mathrm{ng}$ of lambda DNA was fragmented by sonication to 200-300 bp with Covaris S220, followed by end repair and adenylation. Cytosine-methylated barcodes were ligated to sonicated DNA as per the manufacturer's instructions. Thereafter, these DNA fragments were treated twice with bisulfite using EZ DNA Methylation-Gold ${ }^{\mathrm{TM}}$ Kit (Zymo Research, CA, USA) before the single-strand DNA fragments were amplified by PCR using KAPA HiFi HotStart Uracil + ReadyMix (2X). Library concentration was quantified by Qubit $^{\circledR} 2.0$ Fluorometer (Life Technologies, CA, USA) and quantitative PCR, and the insert size was assayed on an Agilent Bioanalyzer 2100 system. The library preparations were sequenced on an Illumina Hiseq 2500/4000 platform, and 125-bp/150-bp paired-end reads were generated. Image analysis and base calling were performed with an Illumina CASAVA pipeline and, finally, 125-bp/150-bp paired-end reads were generated.

\section{Quality control and mapping}

First, FastQC (fastqc_v0.11.5) (Andrews 2010) was used to perform basic statistics relevant to the quality of the raw reads. Thereafter, reads produced by the Illumina pipeline in FASTQ format were preprocessed through Trimmomatic (Trimmomatic-0.36) software (Bolger et al. 2014) using the parameter (SLIDINGWINDOW: 4:15; LEADING: 3, TRAILING: 3; ILLUMINACLIP: adapter.fa: 2: 30: 10; MINLEN: 36 ). The remaining reads that passed all filtering steps were counted as clean reads and used in all subsequent analyses. Finally, FastQC was used to perform basic statistics on the quality of clean data reads.

Bismark software (Felix and Andrews 2011) was used to perform alignments of bisulfite-treated reads to a reference genome (Zhang et al. 2012) using the parameter (-score_min L, 0, -0.2, -X 700 -dovetail). The reference genome was first transformed into a bisulfite-converted version (C-to-T and G-to-A converted) and then indexed using bowtie2 (Langmead and Salzberg 2012). Sequence reads were also transformed into fully bisulfite-converted versions (C-to-T and G-to-A converted) before they were aligned to similarly converted versions of the genome in a directional manner. Sequence reads that produced a unique alignment from the two alignment processes (original top and bottom strand) were then compared with the normal genomic sequence, and the methylation state of all cytosine positions in the read was inferred. The same reads that aligned to the same regions of genome were regarded as duplicates. The sequencing depth and coverage were summarized using deduplicated reads. The bisulfite nonconversion rate was calculated as the percentage of cytosine sequenced at cytosine reference positions in the lambda genome.

\section{Methylation-level calculation and differentially methylated region analysis}

To calculate the methylation level (ML) of the sequence, sequences were divided into multiple bins, with a bin size of $10 \mathrm{~kb}$. The sum of methylated and unmethylated read counts in each window was calculated. ML was defined as

$$
\operatorname{ML}(C)=\frac{\operatorname{reads}(m C)}{\operatorname{reads}(m C)+\operatorname{reads}(C)} .
$$

Calculated ML was further corrected with the bisulfite nonconversion rate according to previous studies (Lister et al. 2013). Given the bisulfite nonconversion rate $r$, the corrected ML was estimated as

$\mathrm{ML}($ corrected $)=\frac{\mathrm{ML}-r}{1-r}$.

Differentially methylated regions (DMRs) were identified using DSS software (Hao et al. 2014; Hao et al. 2015; Park and $\mathrm{Wu}$ 2016) with parameters (smoothing.span $=$ 200 , delta $=0$, p.threshold $=1 \mathrm{e}-05$, minlen $=50, \operatorname{minCG}=$ 3 , dis.merge $=100$, pct.sig $=0.5$ ). According to the distribution of DMRs through the genome, we defined the differentially methylated genes (DMGs) as genes whose gene body region (from transcription start site to transcription end site) or promoter region (from $2 \mathrm{~kb}$ upstream to the transcription start site) had at least 1-bp overlap with the DMRs.

\section{SNP identification and correlation analysis}

SNP calling was conducted using Bis-SNP v.1.0.1 software (Liu et al. 2012) individually $(n=12)$ on deduplicated BAM files produced from the Bismark pipeline, with 5.74 million positions generated. High-quality SNP positions 
were filtered at the coverage of 10-100 in each sample, with 2.32 million positions $(40.42 \%)$ remaining. Remained SNPs were used to perform pairwise genotypic correlations.

To evaluate the genetic effect on epigenetic difference between the two tidal oysters, we used sample groups of $\mathrm{InC}$ and SubC to calculate $F_{\mathrm{ST}}$ and conduct correlation analysis with methylation level according to Liew et al.'s method (Liew et al. 2020). A total of 16,516 genes passed the filtration with $\geq 10$ SNPs and $\geq 5$ methylated positions. $F_{\mathrm{ST}}$ value in per position was calculated using the equation described in Bhatia et al. (Bhatia et al. 2013)

$\widehat{F}=\frac{\sum_{k=1}^{M} \widehat{N}^{[k]}}{\sum_{k=1}^{M} \widehat{D}^{[k]}}$

where $F$ is the averaged $F_{\text {ST }}$ value across a particular gene and markers $k=1,2, \ldots, M$ denote individual SNPs within this gene. $N$ and $D$ are the numerator and denominator of Hudson's equation, respectively

$$
\begin{aligned}
& N=\left(p_{1}-p_{2}\right)^{2}, \\
& D=p_{1}\left(1-p_{2}\right)+p_{2}\left(1-p_{1}\right),
\end{aligned}
$$

where $p_{1}$ and $p_{2}$ denote allelic frequencies of the nonreference base among the intertidal and subtidal samples, respectively.

\section{DAPC analysis}

A discriminant analysis of principal components (DAPC) was conducted using methylation level of genes calculated on common C sites among 12 samples in order to remove genetic bias in adegenet $R$ package (Jombart et al. 2010). To compare plasticity of DNA methylation in response to heat stress, we used the unstressed oyster $(0 \mathrm{~h})$ axis as a measuring scale to quantify the shift in DNA methylation in oysters following heat stress. The magnitude of this shift represents a quantitative measure of genome-wide methylation plasticity. We inferred the size of the shift at heat shock by comparing with that at $0 \mathrm{~h}$ (the length of the arrows in Fig. 1) using Markov Chain Monte Carlo (MCMC) linear mixed models. We derived the $P$ value for plasticity difference of intertidal and subtidal samples by analyzing 2800 MCMC samples of parameter estimates.

\section{Association between DNA methylation and gene expression}

We used the RNA-seq data produced by our previous study (Li et al. 2018), which are publicly available at the Sequence Read Archive database with the accession number
PRJNA407831, and matched it with the WGBS data on identical materials from the present study. To analyze the correlation between DNA methylation and expression level, the mean methylation level and fragments per kilobase million (FPKM) value for each gene was calculated. Scatterplot and Pearson correlation coefficients $(r)$ were performed to display the global correlation between methylation level in gene body and expression. Genes were further grouped together according to expression level to exhibit the corresponding methylation status. Nonexpressed genes $(\mathrm{FPKM}<1)$ and expressed genes were grouped. The latter were then divided into three groups, with the first quartile $(\mathrm{FPKM}<25 \%)$ having the lowest expression level and the last quartile (FPKM $>75 \%)$ having the highest.

\section{Enrichment analysis of DMR-related genes}

Gene Ontology (GO) enrichment analysis of genes related to DMRs was implemented by the GOseq $\mathrm{R}$ package (Young et al. 2010), in which gene-length bias was corrected. GO terms with corrected $P<0.05$ were considered significantly enriched by DMR-related genes. To understand the high-level functions and utilities of the biological system, pathway analysis and statistical testing was conducted using Kyoto Encyclopedia of Genes and Genomes (KEGG) enrichment by means of KOBAS software (Mao et al. 2005).

\section{Data validation by traditional bisulfite-sequencing PCR}

Traditional bisulfite-sequencing PCR (BSP) was used to validate the WGBS data. The mixed DNA used in the WGBS was converted with bisulfite and purified according to EpiTect Fast DNA Bisulfite Kit (QIAGEN, Dusseldorf, Germany) instructions. PCR was carried out on bisulfitetreated DNA using primers specific to the converted DNA sequence (forward primer sequence: TTTTGGATTTTG GAATAATATATTT; reverse primer sequence: ACA TATCCACTATTTTAAAAAAACTTAA). The reference sequence (scaffold1801:18940-19215 at CGI_10004348) was downloaded from the NCBI GenBank database (GenBank: JH816177.1). BSP was performed with $2 \times$ Vazyme Lamp Master Mix (Vazyme Biotech, Nanjing, China), with the program at $94{ }^{\circ} \mathrm{C}$ for $3 \mathrm{~min}$, then 35 cycles at $94{ }^{\circ} \mathrm{C}$ for $30 \mathrm{~s}, 43{ }^{\circ} \mathrm{C}$ for $30 \mathrm{~s}, 72{ }^{\circ} \mathrm{C}$ for $60 \mathrm{~s}$, and a final extension at $72{ }^{\circ} \mathrm{C}$ for an additional 5 -min period. The amplicons were cloned into a pEASY-T1 vector. Twenty clones for each sample were randomly selected to sequence. Unmethylated lambda DNA was used to confirm bisulfite-conversion efficiency. 
a

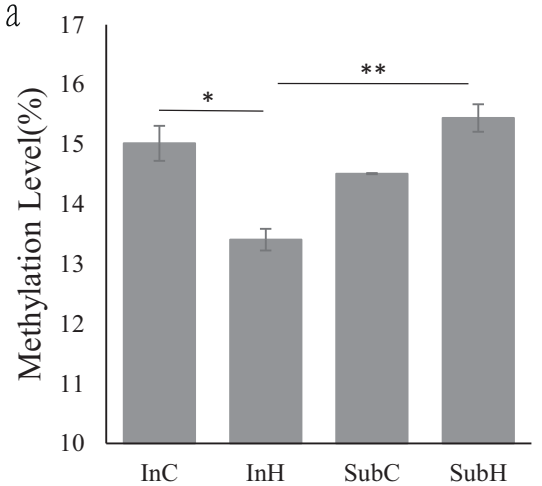

C

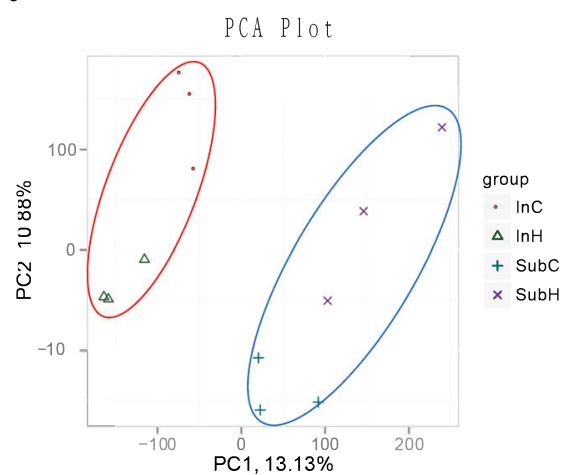

Fig. 1 Global methylation landscape. a Global methylation levels of CpGs of each group. Significant differences calculated from the three biological replicates are indicated by $* P$ value $<0.05$ and $* * P$ value $<$ 0.01 . b Clustering dendrogram of the 12 samples. The red frame denotes the clustered intertidal samples. $\mathbf{c}$ Principal component analysis

d
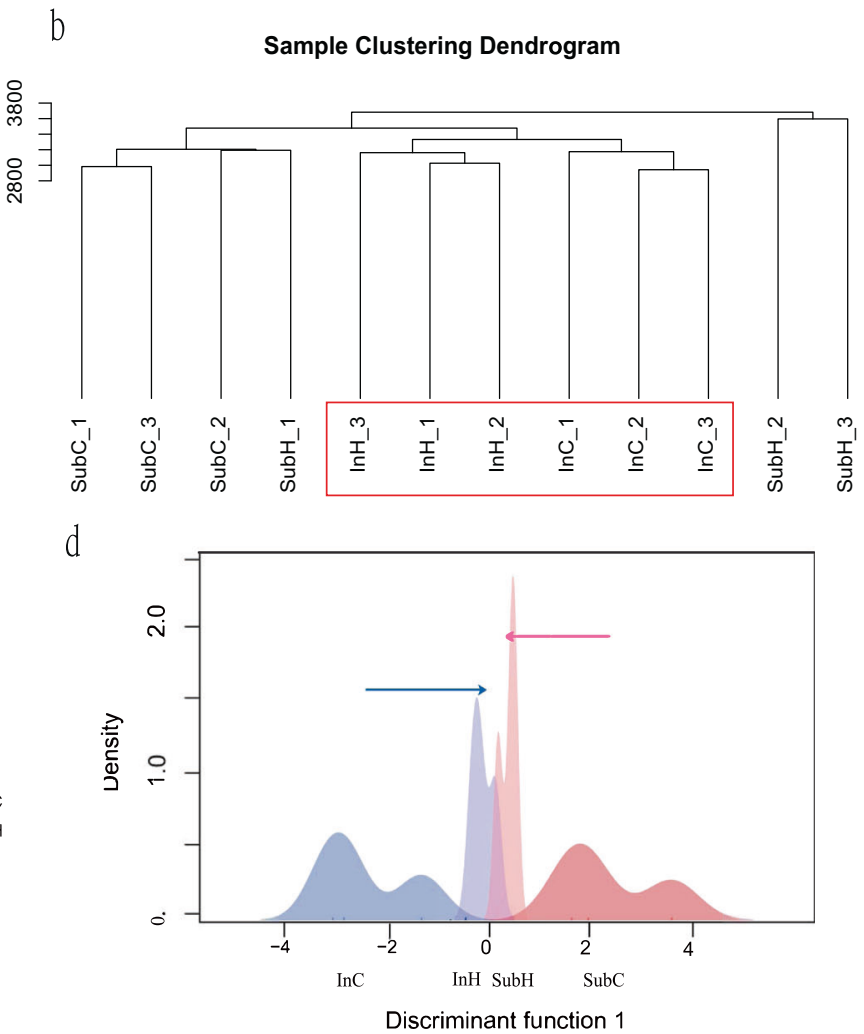

of the 12 samples; the red and blue circles denote the clustered intertidal and subtidal samples. d Variation in DNA methylation plasticity between intertidal and subtidal oysters. The mean methylation changes from control to heat shock in intertidal/subtidal oysters was indicated by the red/blue arrows.
Table 1 Summary of genome-wide methylationsequencing data.

\begin{tabular}{lllllll}
\hline & Raw reads & Clean reads & $\begin{array}{l}\text { Mapped } \\
\text { reads }\end{array}$ & $\begin{array}{l}\text { BS conversion } \\
\text { rate }(\%)\end{array}$ & $\begin{array}{l}\text { Mapping } \\
\text { rate }(\%)\end{array}$ & $\begin{array}{l}5 \times \text { coverage } \\
(\%)\end{array}$ \\
\hline InC & 83021662 & 81275225 & 23332489 & 99.89 & 28.82 & 54.17 \\
InH & 69956461 & 68499401 & 21269426 & 99.89 & 30.99 & 53.90 \\
SubC & 75452051 & 73922747 & 21949575 & 99.88 & 29.87 & 53.59 \\
SubH & 79864515 & 78227942 & 24996679 & 99.88 & 31.99 & 54.31 \\
\hline
\end{tabular}

InC/InH intertidal control/intertidal heat-shock group, SubC/SubH subtidal control/subtidal heatshock group.

\section{Statistical analysis}

Data related to methylation level were presented as mean \pm standard error of mean, and were examined for normality by the Kolmogorov-Smirnov test and for homogeneity by the Levene test. Significant differences in methylation level among the four groups were determined by analysis of variance (ANOVA). Principal component analysis was performed to explore the pattern of methylation level using the function princomp. Last, hierarchical cluster analysis was performed using the pheatmap package in $\mathrm{R}$ software.

\section{Results}

\section{Global methylation pattern of different tidal oysters}

Sequencing of the samples yielded 77 million reads on average, and 23 million of these reads (30\%) from the library were mapped after quality control and alignment. Approximately $54 \%$ of the total clean reads had $>5 \times$ coverage across the genome (Table 1). The details of coverage in 12 samples were listed in Table S1. In the control groups, on average, $15.0 \%$ and $14.5 \%$ of CpGs were methylated in intertidal and subtidal oysters, 
respectively. InH showed significantly lower global methylation levels $(13.4 \%)$ than $\operatorname{InC}(P<0.05)$, whereas SubH exhibited a slightly higher methylation level (15.4\%) than SubC (Fig. 1a). In addition, on average, $0.20 \%$ of $\mathrm{CHG}$ and $0.16 \%$ of $\mathrm{CHH}$ were methylated in control oysters. In this study, methylation in $\mathrm{CpG}$ motifs was only investigated, if not particularly indicated. As shown in Fig. 1b, samples did not cluster according to the heat-stress treatment, but rather by their habitat tidal levels. The methylation profiles obtained from intertidal and subtidal samples clustered, except two of the subtidal samples under heat stress, which diverged from the others. The divergence between the two tidal-level samples can be easily recognized from the PC1 cluster (Fig. 1c). Furthermore, PC2 reflected the differentiation of heat stress and control conditions, with intertidal and subtidal samples displayed in an inverted position. DAPC plot (Fig. 1d) also showed clear differentiation between intertidal and subtidal oysters in response to heat shock. The intertidal oysters presented lower methylation under heat shock, while subtidal oysters showed the opposite. Significantly, differentiation of the shift response to heat shock was detected between the two tidal oysters (MCMC $P<0.05)$.

\section{Estimation of genotypic effect on methylation diversity}

To access the genetic influence on methylation, we identified SNPs from WGBS reads, and calculated the correlation between genotype and epigenotype according to the method proposed by Liew et al. (2020). Pairwise genotypic correlation analysis showed that oysters from the same tidal clustered, indicating that they are more genetically similar (Fig. 2a). In order to investigate the genetic effect on methylation, the correlation between $F_{\mathrm{ST}}$ and methylationlevel difference of the two tidal oysters was examined
(Fig. 2b). Our results showed that there was very weak correlation in gene body $(r=0.02)$ and exon $(r=0.01)$ regions, suggesting that the epigenetic diversity we detected in the two tidal oysters was mostly independent of genotypic.

\section{Correlation between DNA methylation status and gene expression levels}

Figure $3 \mathrm{a}$ shows a positive correlation between methylation level and gene expression in the four groups. In oysters, the methylated fraction tends to consist of gene bodies, while other genomic regions exhibit less methylation (Fig. 3b). Figure $3 \mathrm{~b}$ exhibits similar trends, with nonexpressed genes showing the lowest methylation levels, high- and mediumexpression groups had relatively high methylation levels, and the low-expression group was intermediate between them. The four groups (InC, $\mathrm{InH}, \mathrm{SubC}$, and $\mathrm{SubH}$ ) in this study showed consistent trends. Figure $3 \mathrm{~b}$ only depicts the results of one sample ( $\mathrm{InC})$, and the others were displayed in Fig. S2.

\section{DNA methylation differentiation between oysters at two tidal levels under temperate conditions}

To identify evolved divergence in DNA methylation, DMGs between InC and SubC were identified, and functional enrichment results were exhibited in Fig. S3. Here while we focus on the potential transcriptional consequences of DNA methylation, we examined the overlap genes of DMGs and different expression genes (DEGs). We tested DMGs in all contexts (CG, $\mathrm{CHG}$, and $\mathrm{CHH}$ ) for integral results. There were 90 (11.46\% of DEGs) genes that overlapped with DEGs and DMGs of InC vs. SubC (Fig. 4a). The differentiation of methylation and expression level of these overlap genes is exhibited in a heatmap (Fig. 4b). We performed GO analysis on these genes; significantly enriched terms of biological process were
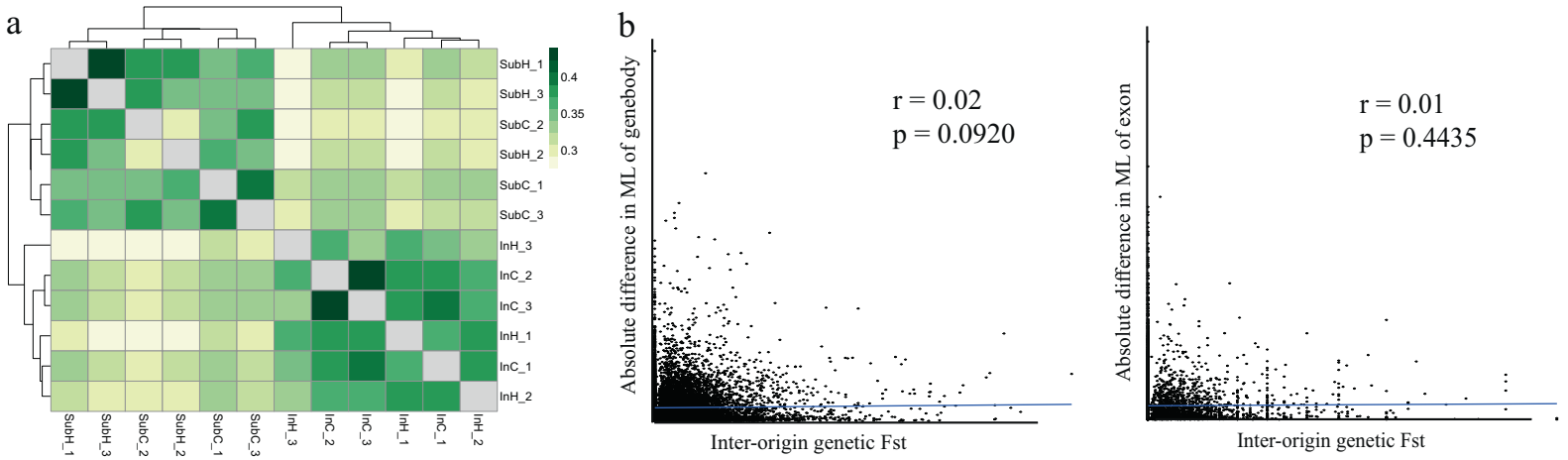

Fig. 2 Assessment of genetic effect on DNA methylation. a Pairwise correlation analysis and clustering of SNP data from all 12 samples. b Scatterplot of $F_{\mathrm{ST}}$ values and methylation-level difference in genes of InC and SubC oysters. 
a

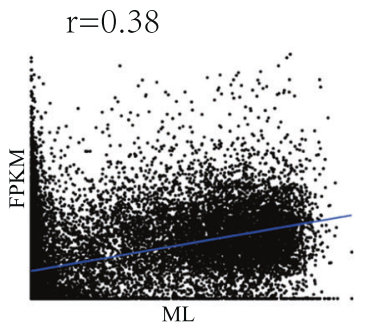

SubC

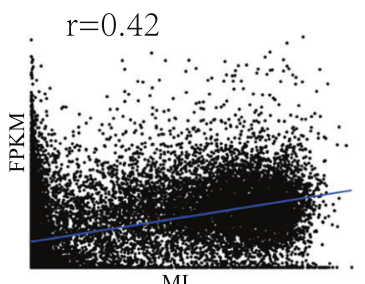

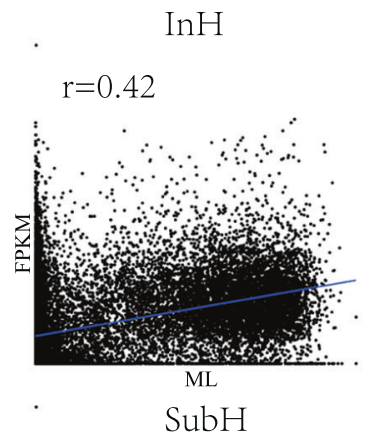

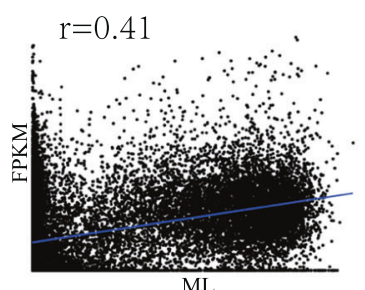

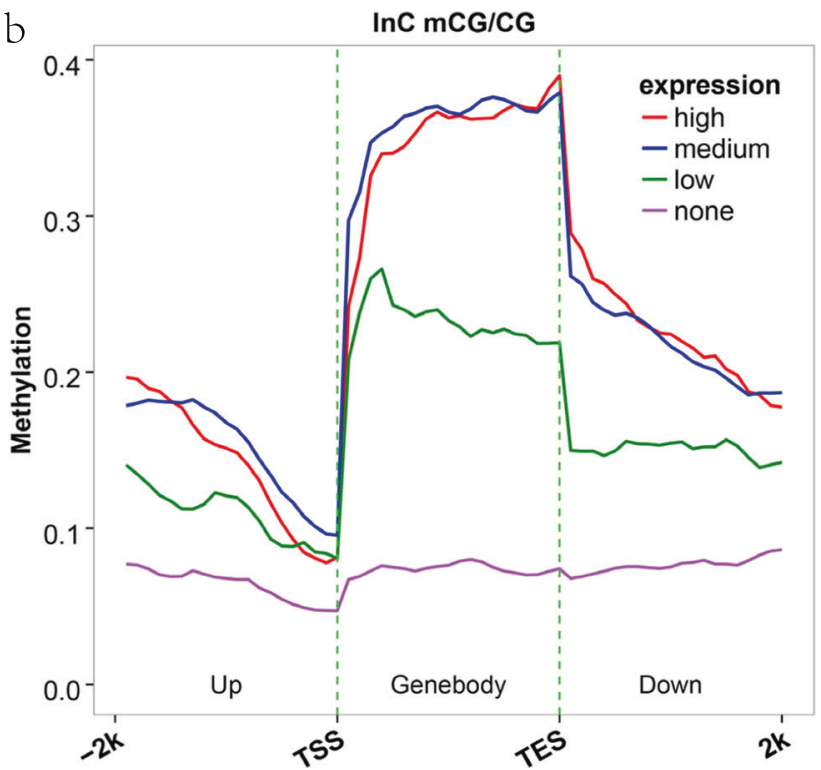

Fig. 3 Relationship between DNA methylation and gene expression. a Correlation analysis of methylation level and expression in genes. b Distributions of methylation levels partitioned by different expression levels. Genes with FPKM value $<0.1$ were considered nonexpressed.

Fig. 4 Analysis of different genes in two tidal oysters. a Venn plot of overlapped genes of DEG and DMG in InC vs. SubC. b Heatmap of methylation level and FPKM in overlapped genes in InC and SubC. c Enriched GO terms of overlapped genes in biological process.
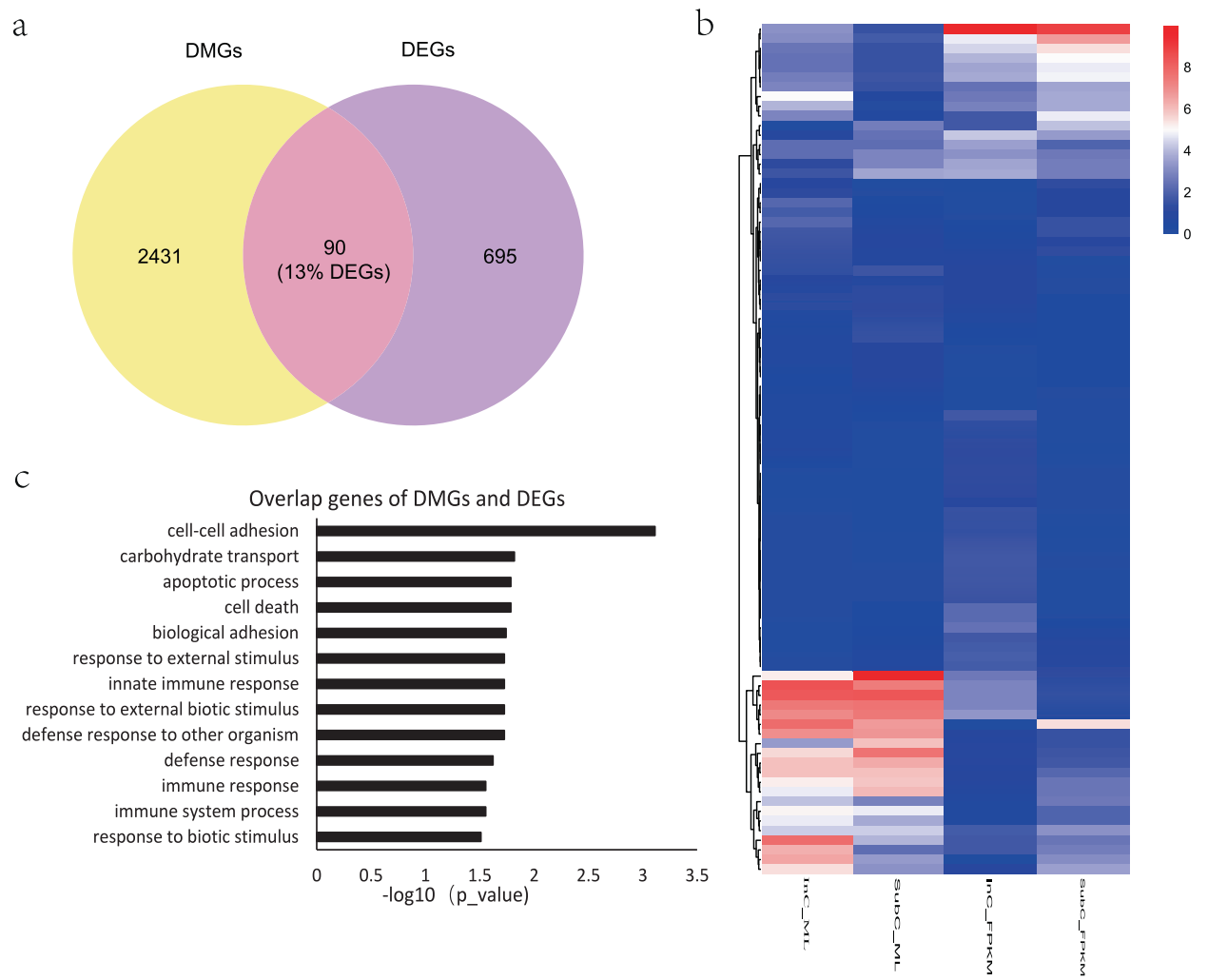

displayed. As shown in Fig. 4c, genes involved in cell-cell adhesion, carbohydrate transport, the apoptotic process, and cell death were included. Among these, six genes were identified and annotated as heat-shock protein (HSP)related genes, with different methylation and expression variations (Table S2).

\section{DNA methylation differentiation between intertidal and subtidal oysters in response to heat stress}

We further compared the methylation variation under heat stress at the whole-genome level, genomic regions, and DMRs. As shown in Fig. 5a, the methylation level of most 
a
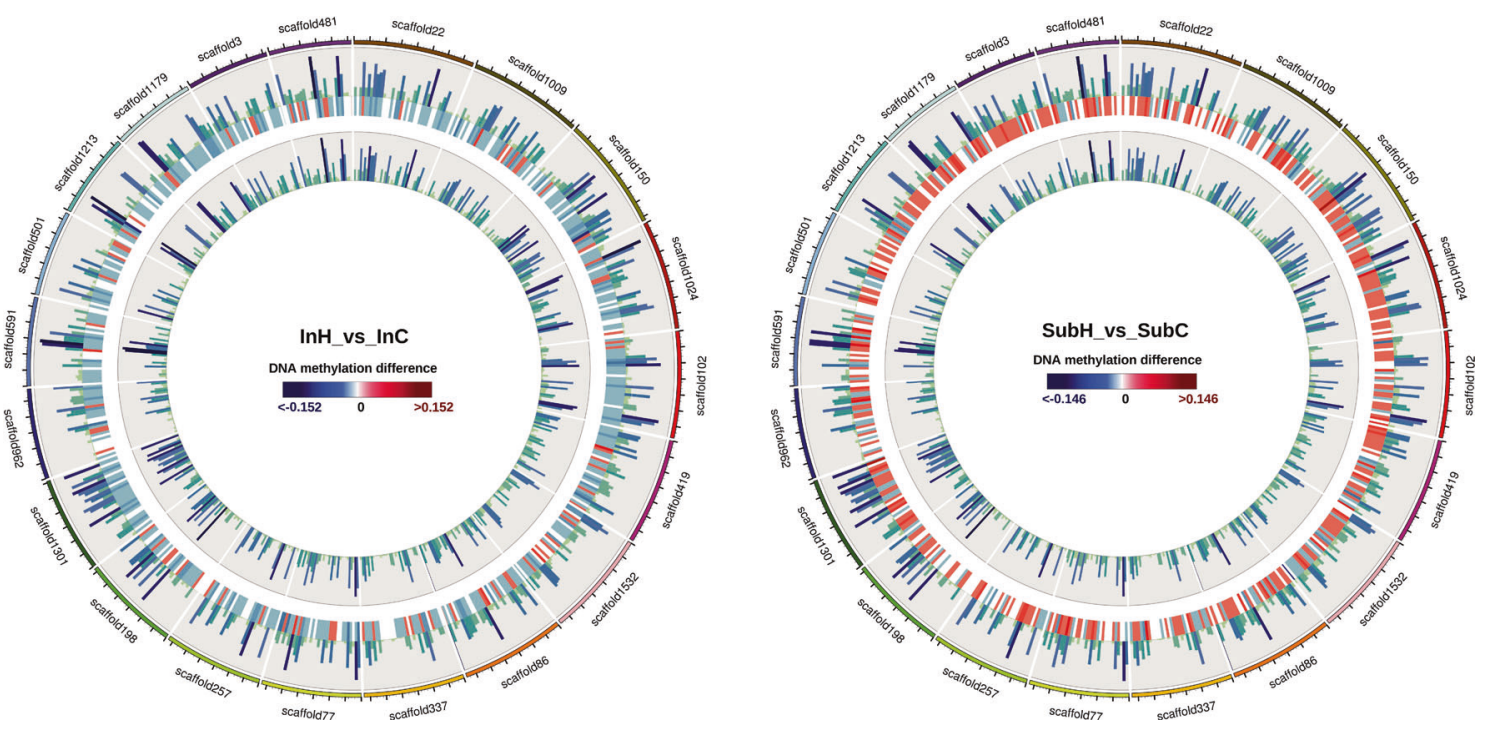

b
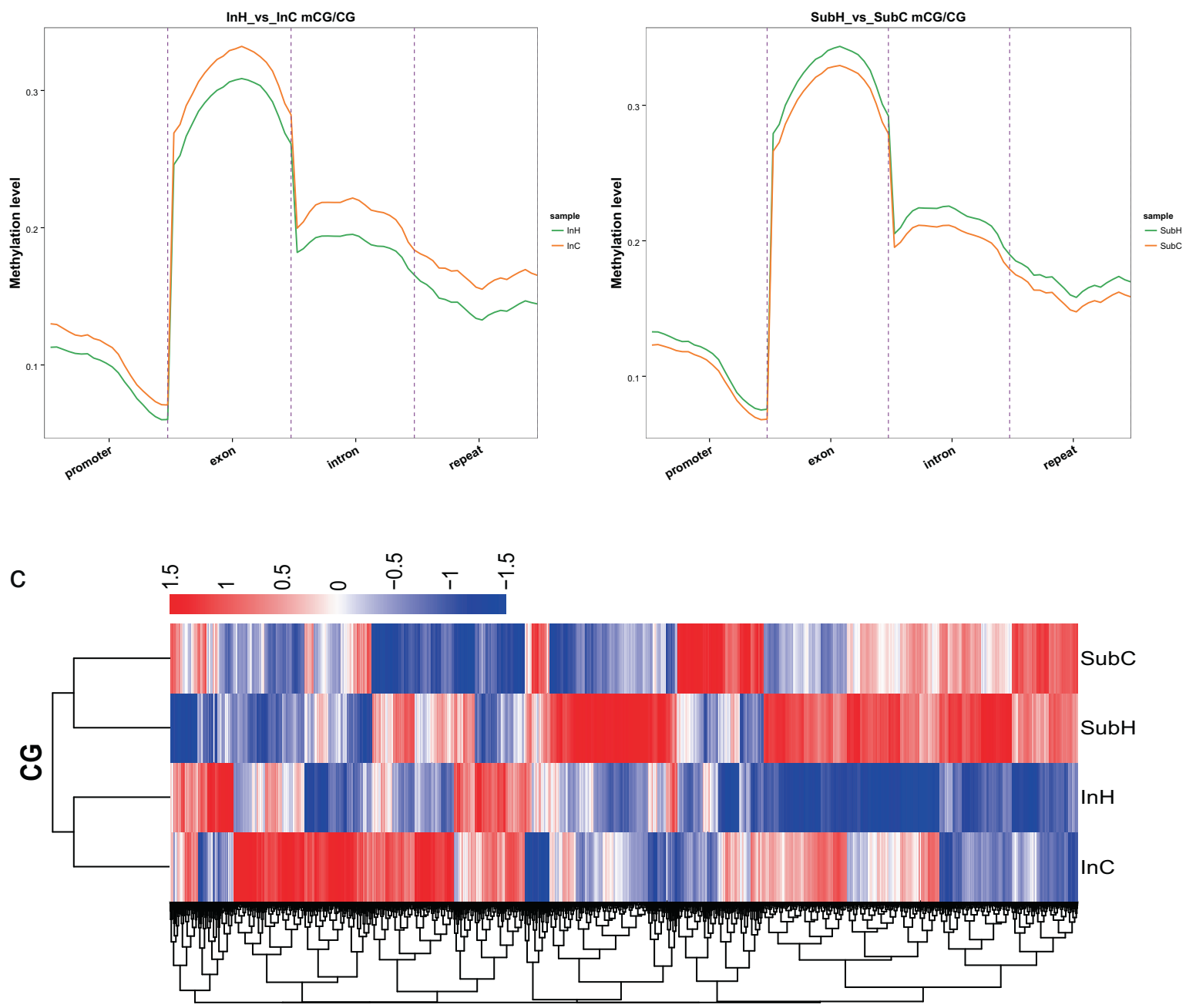

Fig. 5 Comparative methylome analysis under heat shock. a Circos plots of genome scaffolds. The three circles from outer to inner represented the methylation levels of the heat-stress-treated groups (InH and $\mathrm{SubH}$ ), the differences between heat-shock and control groups (InH vs. InC and SubH vs. SubC), and the control groups (InC and SubC), respectively. The level of methylation is denoted by the darkness of the color, and the degree of the difference is represented by the heatmap. b Distributions of DNA methylation levels among different genomic regions. c Heatmap of methylation levels within different methylation regions. Darker red indicates greater methylation in an individual for that DMR, whereas darker blue indicates lesser methylation in an individual for that DMR. 

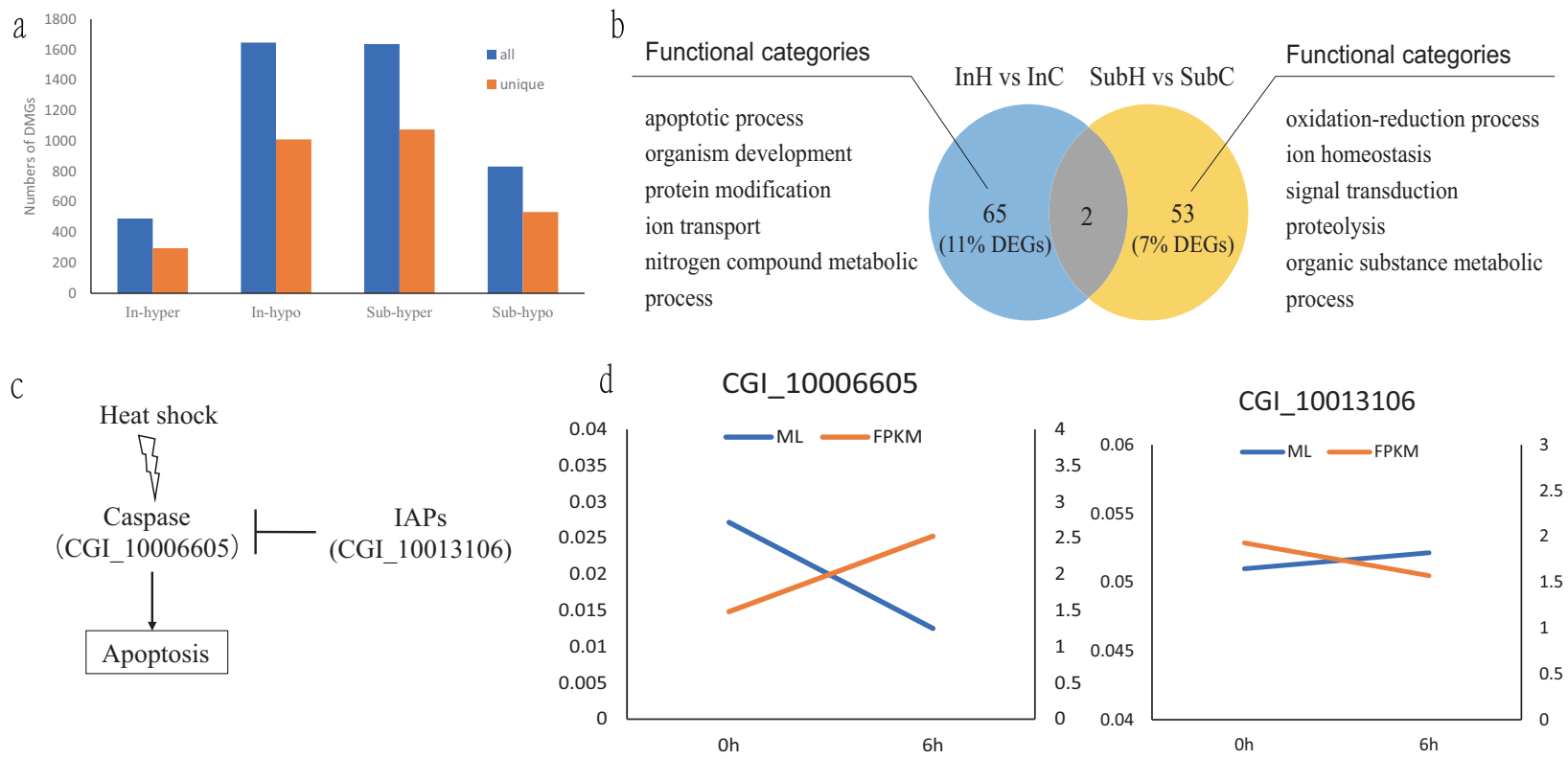

Fig. 6 Analysis of gene response to heat shock. a Numbers of hyperand hypo-DMGs of InH vs. InC and SubH vs. SubC. Unique DMGs represent all DMGs in InH vs. InC, or SubH vs. SubC subtract the common genes between InH vs. InC and SubH vs. SubC. b Venn plot

of the common genes of DMGs and DEGs in InH vs. InC and SubH vs. SubC. Functional categories showed the specific genes enriched in InH vs. InC and SubH vs. SubC. c A simple sketch of apoptosis. d ML and FPKM variations of two genes involved in apoptosis.

$\mathrm{CpG}$ sites in $\mathrm{InH}$ was lower than those of InC, denoted by the blue middle circle for intertidal samples. In contrast, the methylation level of SubH was higher than that of SubC, denoted by the red middle circle. Similar patterns of methylation following heat stress were revealed in the different genomic regions of the promoter, exon, intron, and repeat (Fig. 5b), and in DMRs (Fig. 5c). The heatmap (Fig. 5c) presented all DMRs among the four groups. Oysters from the same vertical level (intertidal or subtidal) were clustered.

There were 491 hypermethylated and 1646 hypomethylated DMGs in InH vs. InC, and 1636 hypermethylated and 823 hypomethylated DMGs in SubH vs. SubC. In total, 788 DMGs were shared between the two groups, whereas 1279 and 1554 DMGs varied uniquely in intertidal and subtidal samples, respectively, when exposed to high temperatures (Fig. 6a). The column chart exhibits the different methylation patterns between intertidal and subtidal samples. Enrichment results of GO and KEGG pathways were supplemented in Fig. S4.

Combined with the transcriptional data, 67 (10.65\% of DEGs) and 55 (6.88\% of DEGs) overlap genes were found in $\mathrm{InH}$ vs. InC and SubH vs. SubC, respectively (Fig. 6b). The relationship between methylation and expression level of the specific genes in $\mathrm{InH}$ vs. InC and $\mathrm{SubH}$ vs. SubC (Fig. S5) is displayed as a scatterplot. Many of the genes in the intertidal group showed hypomethylation (77\%), and $42 \%$ of genes showed downregulated expression. In contrast, genes showing hypermethylation combined with upregulated expression accounted for $62 \%$ of the genes in

the subtidal group. Taken together, a positive correlation was observed between DNA methylation and gene expression, which is in accordance with the trend at the whole-genome level (Fig. 3a, b).

Only two genes were common to both groups. One is a component of the HSP gene (CGI_10004348), which encodes heat-shock $70-\mathrm{kDa}$ protein 12A (HSPA12A), and showed upregulated expression in the two groups under heat shock. However, the methylation varied: intertidal samples showed a positive relationship with expression, whereas subtidal samples showed the opposite (Fig. S6). To validate the WGBS data, a target area containing eight CpGs (276 bp, scaffold1801:18940-19215) on HSPA12A was selected according to the results shown in Integrative Genomics Viewer (Fig. S7a) to carry out BSP. The bisulfite-conversion rates represented by the percentage of unmethylated lambda DNA were over 99\%. As shown in Fig. S7b, InC was almost unmethylated within the eight CpGs, while InH exhibited higher methylation levels. Similarly, SubH showed higher methylation levels than SubC. These results indicated that the traditional bisulfitesequencing results were in good accordance with the WGBS data. The function of the other common gene (CGI_10002343) was not annotated, and both the expression and methylation level of this gene decreased in the two groups when faced with high temperatures (Fig. S6).

Specific genes in InH vs. InC were found to participate in regulation of the apoptotic process, organism development, protein modification, and ion transport. As for subtidal-specific genes, functional categories were enriched 
in the oxidation-reduction process, ion homeostasis, signal transduction, and proteolysis (Fig. 6b). Details of the relevant genes are listed in Tables S3 and S4. Among the genes in $\mathrm{InH}$ vs. InC, two were found to participate in the apoptotic process. One (CGI_10006605) is annotated as a caspase-like protein, the other (CGI_10013106) is a member of the inhibitor of apoptosis protein (IAP) family (Fig. 6c). Caspase activation plays a central role in the execution of apoptosis, but IAP is an inhibitor of apoptosis. CGI_10006605 showed hypomethylation and upexpression, whereas CGI_10013106 showed hypermethylation and downexpression (Fig. 6d). Both of these changes in expression contributed to apoptosis, which was negatively regulated by DNA methylation.

\section{Discussion}

A growing number of studies have shown that a dynamic environment can lead to changes in epigenetic marks, which may influence phenotypic plasticity, and help explain the mechanisms of rapid adaptation and acclimatization in a future of rapidly changing climate (Metzger and Schulte 2017; Putnam et al. 2016; Zhang et al. 2017a). Our previous study revealed the phenotypic divergence between intertidal and subtidal oysters (Li et al. 2018). In this study, divergent methylation patterns were revealed between intertidal and subtidal oysters, and the genes potentially regulated by the methylation were identified. This study dissected the heritable component of environment-induced phenotypic variation.

The methylation landscape of $C$. gigas was investigated in this research. Approximately $15 \%$ of CpGs were identified methylated in this study. This result is in accordance with previous studies in C. gigas using WGBS (Gavery and Roberts 2013; Olson and Roberts 2014). Compared with other invertebrates, this level is lower than that reported in the sea squirt (Halocynthia roretzi, 29\%), but higher than that in sea anemones (Nematostella vectensis, 11\%) (Zemach et al. 2010). Heat shock can cause significantly decreased and slightly increased methylation levels in intertidal and subtidal oysters, respectively, indicating that DNA methylation can be induced by acute heat shock. This result is supported by previous studies. For example, high temperature can induce significant global DNA methylation changes in Didemnum vexillum (Hawes et al. 2018), and any change in temperature during development and thermal acclimation in adults can increase DNA methylation levels in Gasterosteus aculeatus (Metzger and Schulte 2017). Consistent with previous research on oysters (Gavery and Roberts 2013), this study found that DNA methylation tended to be mainly located in gene bodies, with other genomic regions exhibiting less methylation.
As for the relationship between methylation and expression, we found that highly and intermediately expressed genes exhibited the highest degree of methylation in gene bodies. This observation is in agreement with previous studies in the oyster (Gavery and Roberts 2013; Olson and Roberts 2014), the honeybee (Lyko et al. 2010), eukaryotes (Zemach et al. 2010), and mammals (Aran et al. 2011), indicating that the association of hypermethylation in gene bodies with higher expression appears to be a general phenomenon. The occurrence of gene body methylation, especially in protein-coding sequences, was found that can cause deleterious amino acid substitutions (Dixon et al. 2016); to compensate this risk, it was reported that gene body methylation can be altered by environmental factors, and thus balances the expression of housekeeping and environmentally responsive genes to help acclimatization (Dixon et al. 2018). The divergence of phenotypic plasticity in gene expression in oysters has been investigated in a previous study, in which intertidal oysters showed higher plasticity than subtidal oysters (Li et al. 2018). In accordance with this, our research on DNA methylation showed that intertidal oysters were more plastic, which implied that DNA methylation may mediate adaptive phenotypic plasticity.

Oysters derived from intertidal and subtidal areas showed epigenetic divergence. In this study, genotype showed limited influences on epigenetic differentiation between the two tidal oysters, suggesting that the methylation variation we detected was mainly induced by environment. The results of sample clustering showed that the differentiation of DNA methylation between the two groups was greater than that induced by acute heat shock, which emphasized that the mediating role of DNA methylation was strongly influenced by life history in specific environments. This phenomenon is not unexpected, considering the mechanism of DNA methylation building, which allows the stable reproduction of gene-activity states from a parental generation of cells to the next. The epigenetic modification we detected in the oyster could be a quantitative response, since the environment-induced epigenetic effect depends on the magnitude of the affecting factor, such as long-term life history or acute heat shock (Jaenisch and Bird 2003). Previous studies reported that oysters inhabiting intertidal areas are considered more heat-tolerant, whereas individuals living in subtidal areas are more heat-sensitive (Diederich and Pechenik 2013; Li et al. 2018). In this study, intertidal oysters mainly showed genomic hypomethylation, while subtidal samples displayed hypermethylation, in response to heat shock. Interestingly, a similar reverse methylation pattern was also detected in a study of two genotypes of rapeseed seedlings, which found that more DNA demethylation events occurred in the heat-tolerant genotype, while more methylation events occurred in the heat-sensitive genotype (Gao et al. 2014). The reverse methylation pattern 
implies that the epigenetic differentiation between the two groups of oysters in this study may result from genetic divergence, which could have resulted from post-settlement mortality when oyster larvae settled onshore, although there is a strong gene flow between intertidal and subtidal oysters at the same geographical location (Sanford and Kelly 2011; Schmidt and Rand 2001).

The extent of the role of DNA methylation in regulating gene expression may be demonstrated by $10.65 \%$ and $6.88 \%$ DMGs in DEGs in intertidal and subtidal oysters, respectively. Oysters derived from two tidal areas modulate totally different genes in response to heat shock, as intertidal oysters were engaged in apoptosis and organism development processes, while oxidationreduction and ion homeostasis processes occurred in subtidal oysters. These results indicated that the two groups of oysters were experiencing varied response status, as found by Li et al. (2018), who demonstrated different thermal adaptation in different geographical $C$. gigas populations. Two affected genes related to promotion of the apoptotic process can contribute to thermal response in the intertidal samples, because studies on corals have demonstrated the involvement of apoptosis in response to rising seawater temperature, and suggested that increasing temperatures can activate caspasedependent apoptosis in irreparable cells to improve organismal changes of survival (Kvitt et al. 2016; Pernice et al. 2011). The expression of both genes was negatively correlated with DNA methylation, from which can be inferred that DNA methylation can suppress gene expression by inhibiting the binding of transcription factors to DNA, or by recruiting transcriptional repressor proteins (Moore et al. 2013). In support of this observation, a negative correlation between DNA methylation and expression of some homeobox gene orthologs during early oyster development has also been reported (Riviere et al. 2013). Overall, apoptotic response may play an important role in intertidal adaptation, and the DNA methylation we detected provides a new underlying mechanism.

Several genes in the HSP family were found in both intertidal and subtidal groups. A previous study reported DNA hypomethylation of the Biomphalaria glabrata HSP 70 region in snails under heat shock (Ittiprasert et al. 2015). However, our data showed that both hyper- and hypomethylation occur, combined with both increased and decreased expression. This result may be explained by the different habitats and treatments of the organisms. Remarkably, the gene encoding HSPA12A was the only annotated gene that showed significant methylation and expression variation in both intertidal and subtidal oysters. It has been demonstrated to participate in environmental stress-response processes by regulating gene expression in a variety of organisms, such as mussels, mice, and Japanese black cattle (Han et al. 2003; Masoudi et al. 2008; You et al. 2013; Zhu et al. 2013). However, little is known regarding how DNA methylation works on HSPA12A when under environmental stressors. Hence, our results not only complement the existing literature on the role of epigenetics in this gene in response to thermal stress, but also reveal the different regulatory patterns in two regional oysters.

In this study, heritable epigenetic variation was investigated by using F1 offspring subjected to common garden experiment. The divergence between the intertidal and the subtidal oysters for the multiple traits clearly suggests that the environment affects phenotypic variation, and that this adaptive phenotypic divergence may be mediated by DNA methylation that can be transmitted to the next generation. Although transgenerational environment-induced epigenetic variants have been revealed in this study, it remains unclear to what extent the epigenetic changes can stably persist across generations, and what mechanism underlies this DNA methylation erasure and re-establishment.

\section{Conclusion}

Understanding the mechanisms regulating the capacity of organisms to respond to environmental variation is critical in determining the impacts of environmental change on populations. In this study, we used WGBS combined with RNA-seq to investigate DNA methylation features in oysters derived from different tidal areas. Our data demonstrated that DNA methylation in $C$. gigas can be induced by environmental changes, and differentiation in methylation was detected between intertidal and subtidal oysters. In addition, the genes potentially regulated by methylation were identified. This study revealed that methylation is a mechanism underlying adaptive phenotypic divergence between the intertidal and subtidal oysters. This will facilitate a better understanding of how epigenetic mechanisms participate in environmental stress responses in marine invertebrates, and provides new avenues for resource conservation and management.

\section{Data availability}

The methylome data have been deposited in the Sequence Read Archive database with the accession number PRJNA562805.

Acknowledgements LL is supported by the National Key R\&D Program of China (No. 2018YFD09-00304) and Key Deployment Project of Centre for Ocean Mega-Research of Science, Chinese Academy of Sciences (COMS2019Q06). AL is supported by the Distinguished Young Scientists Research Fund of Key Laboratory of Experimental 
Marine Biology, Chinese Academy of Sciences (No. KLEMB-DYS04). LL is also supported by the National Natural Science Foundation of China (No. 31572620) and the Technology and the Modern Agroindustry Technology Research System (No. CARS-49). We thank professor Manuel Aranda for guidance on SNP calling and related analysis, and thank Dr. Yuxia Zou for advices with BSP experiment.

\section{Compliance with ethical standards}

Conflict of interest The authors declare that they have no conflict of interest.

Publisher's note Springer Nature remains neutral with regard to jurisdictional claims in published maps and institutional affiliations.

\section{References}

Andrews S (2010) Babraham bioinformatics. Babraham Institute, Cambridge, United Kingdom

Aran D, Toperoff G, Rosenberg M, Hellman A (2011) Replication timing-related and gene body-specific methylation of active human genes. Hum Mol Genet 20(4):670-680

Bhatia G, Patterson N, Sankararaman S, Price AL (2013) Estimating and interpreting FST: the impact of rare variants. Genome Res 23 (9):1514-1521

Bolger AM, Lohse M, Usadel B (2014) Trimmomatic: a flexible trimmer for Illumina sequence data. Bioinformatics 30(15):2114-2120

Deans C, Maggert KA (2015) What do you mean, "epigenetic"? Genetics 199(4):887-896

Diederich CM, Pechenik JA (2013) Thermal tolerance of Crepidula fornicata (Gastropoda) life history stages from intertidal and subtidal subpopulations. Mar Ecol-Prog Ser 486:173-187

Dixon G, Liao Y, Bay LK, Matz MV (2018) Role of gene body methylation in acclimatization and adaptation in a basal metazoan. Proc Natl Acad Sci USA 115(52):13342-13346

Dixon GB, Bay LK, Matz MV (2016) Evolutionary consequences of DNA methylation in a basal metazoan. Mol Biol Evol 33 (9):2285-2293

Ellison A, Rodriguez Lopez CM, Moran P, Breen J, Swain M, Megias M et al. (2015) Epigenetic regulation of sex ratios may explain natural variation in self-fertilization rates. Proc Biol Sci 282:1819

Felix K, Andrews SR (2011) Bismark: a flexible aligner and methylation caller for Bisulfite-Seq applications. Bioinformatics 11:11

Fernandez-Reiriz MJ, Irisarri J, Labarta U (2016) Flexibility of physiological traits underlying inter-individual growth differences in intertidal and subtidal Mussels Mytilus galloprovincialis. PLoS ONE 11(2): 0148245

Gao G, Li J, Li H, Li F, Xu K, Yan G et al. (2014) Comparison of the heat stress induced variations in DNA methylation between heat-tolerant and heat-sensitive rapeseed seedlings. Breed Sci 64(2):125-133

Gavery MR, Roberts SB (2013) Predominant intragenic methylation is associated with gene expression characteristics in a bivalve mollusc. PeerJ 1:e215

Guo X, Li Q, Wang QZ, Kong LF (2012) Genetic mapping and QTL analysis of growth-related traits in the Pacific oyster. Mar Biotechnol 14(2):218-226

Han Z, Truong QA, Park S, Breslow JL (2003) Two Hsp70 family members expressed in atherosclerotic lesions. Proc Natl Acad Sci USA 100(3):1256-1261

Hao F, Conneely KN, Hao W (2014) A Bayesian hierarchical model to detect differentially methylated loci from single nucleotide resolution sequencing data. Nucleic Acids Res 42(8):e69
Hao W, Tianlei X, Hao F, Li C, Ben L, Bing Y et al. (2015) Detection of differentially methylated regions from whole-genome bisulfite sequencing data without replicates Nucleic Acids Res 43(21): e141

Hawes NA, Tremblay LA, Pochon X, Dunphy B, Fidler AE, Smith KF (2018) Effects of temperature and salinity stress on DNA methylation in a highly invasive marine invertebrate, the colonial ascidian Didemnum vexillum. Peer J 6:e5003

Holloway GJ (2002) Phenotypic Plasticity: Beyond Nature and Nurture. Heredity 89(6):410

Ittiprasert W, Miller A, Knight M, Tucker M, Hsieh MH (2015) Evaluation of cytosine DNA methylation of the Biomphalaria glabratahe at shock protein 70 locus after biological and physiological stresses. J Parasitol Vector Biol 7(10):182-193

Jaenisch R, Bird A (2003) Epigenetic regulation of gene expression: how the genome integrates intrinsic and environmental signals. Nat Genet 33(Suppl):245-254

Jombart T, Devillard S, Balloux F (2010) Discriminant analysis of principal components: a new method for the analysis of genetically structured populations. BMC Genet 11:94

Kvitt H, Rosenfeld H, Tchernov D (2016) The regulation of thermal stress induced apoptosis in corals reveals high similarities in gene expression and function to higher animals. Sci Rep. 6:30359

Langmead B, Salzberg SL (2012) Fast gapped-read alignment with Bowtie 2. Nat Methods 9(4):357-359

Li A, Li L, Wang W, Song K, Zhang G (2018) Transcriptomics and fitness data reveal adaptive plasticity of thermal tolerance in oysters inhabiting different tidal zones. Front Physiol 9:825

Liew YJ, Howells EJ, Wang X, Michell CT, Burt JA, Idaghdour Y et al. (2020) Intergenerational epigenetic inheritance in reefbuilding corals. Nat Clim Change 10(3):254-259

Liew YJ, Zoccola D, Li Y, Tambutte E, Venn AA, Michell CT et al. (2018) Epigenome-associated phenotypic acclimatization to ocean acidification in a reef-building coral. Sci Adv 4(6):eaar8028

Lira-Medeiros CF, Parisod C, Fernandes RA, Mata CS, Cardoso MA, Ferreira PC (2010) Epigenetic variation in mangrove plants occurring in contrasting natural environment. PLoS ONE 5(4):e10326

Lister R, Mukamel EA, Nery JR, Urich M, Puddifoot CA, Johnson ND et al. (2013) Global epigenomic reconfiguration during mammalian brain development. Science 341(6146):1237905

Liu Y, Siegmund KD, Laird PW, Berman BP (2012) Bis-SNP: combined DNA methylation and SNP calling for Bisulfite-seq data. Genome Biol 13(7):R61

Lyko F, Foret S, Kucharski R, Wolf S, Falckenhayn C, Maleszka R (2010) The Honey Bee Epigenomes: Differential Methylation of Brain DNA in Queens and Workers. PLoS Biol 8(11): e1000506

Mao X, Tao CJGO, Wei L (2005) Automated genome annotation and pathway identification using the KEGG Orthology (KO) as a controlled vocabulary. Bioinformatics 21(19):3787-3793

Marsh AG, Pasqualone AA (2014) DNA methylation and temperature stress in an Antarctic polychaete, Spiophanes tcherniai. Front Physiol 5:173

Masoudi AA, Uchida K, Yokouchi K, Ohwada K, Abbasi AR, Tsuji T et al. (2008) Linkage mapping of the locus responsible for forelimb-girdle muscular anomaly of Japanese black cattle on bovine chromosome 26. Anim Genet 39(1):46-50

Metzger DCH, Schulte PM (2017) Persistent and plastic effects of temperature on DNA methylation across the genome of threespine stickleback (Gasterosteus aculeatus). Proc Biol Sci 284:1864

Mondol MR, Kim C-W, Kang C-K, Park SR, Noseworthy RG, Choi K-S (2016) Growth and reproduction of early grow-out hardened juvenile Pacific oysters, Crassostrea gigas in Gamakman Bay, off the south coast of Korea. Aquaculture 463:224-233

Moore LD, Le T, Fan G (2013) DNA methylation and its basic function. Neuropsychopharmacology 38(1):23-38 
Olson CE, Roberts SB (2014) Genome-wide profiling of DNA methylation and gene expression in Crassostrea gigas male gametes. Front Physiol 5:224

Park Y, Wu H (2016) Differential methylation analysis for BS-seq data under general experimental design. Bioinformatics 32 (10): $1-10$

Pernice M, Dunn SR, Miard T, Dufour S, Dove S, Hoegh-Guldberg O (2011) Regulation of apoptotic mediators reveals dynamic responses to thermal stress in the reef building coral Acropora millepora. PLoS ONE 6(1):e16095

Place SP, Menge BA, Hofmann GE (2012) Transcriptome profiles link environmental variation and physiological response of Mytilus californianus between Pacific tides. Funct Ecol 26 (1):144-155

Pörtner HO, Lloyd P, George S (2007) Thermal limits and adaptation in marine Antarctic ectotherms: an integrative view. Philos Trans R Soc Lond 362(1488):2233-2258

Putnam HM, Davidson JM, Gates RD (2016) Ocean acidification influences host DNA methylation and phenotypic plasticity in environmentally susceptible corals. Evol Appl 9(9):1165-1178

Riviere G, Wu GC, Fellous A, Goux D, Sourdaine P, Favrel P (2013) DNA methylation is crucial for the early development in the Oyster C. gigas. Mar Biotechnol 15(6):739-753

Rondon R, Grunau C, Fallet M, Charlemagne N, Sussarellu R, Chaparro C et al. (2017) Effects of a parental exposure to diuron on Pacific oyster spat methylome. Environ Epigenet 3(1):dvx004

Sanford E, Kelly MW (2011) Local adaptation in marine invertebrates. Ann Rev Mar Sci 3:509-535

Schmidt PS, Rand DM (2001) Adaptive maintenance of genetic polymorphism in an intertidal barnacle: habitat- and life-stage-specific survivorship of Mpi genotypes. Evolution 55(7):1336-1344

Schulte PM, Healy TM, Fangue NA (2011) Thermal performance curves, phenotypic plasticity, and the time scales of temperature exposure. Integr Comp Biol 51(5):691-702

Somero GN (2010) The physiology of climate change: how potentials for acclimatization and genetic adaptation will determine 'winners' and 'losers'. J Exp Biol 213(6):912-920
Southward JA (1958) Note on the temperature tolerances of some intertidal animals in relation to environmental temperatures and geographical distribution. J Mar Biol Assoc UK 37(01):49

Sunday JM, Bates AE, Dulvy NK (2012) Thermal tolerance and the global redistribution of animals. Nat Clim Change 2:686

Venegas D, Marmolejo-Valencia A, Valdes-Quezada C, Govenzensky T, Recillas-Targa F, Merchant-Larios H (2016) Dimorphic DNA methylation during temperature-dependent sex determination in the sea turtle Lepidochelys olivacea. Gen Comp Endocrinol 236:35-41

Williams SE, Shoo LP, Isaac JL, Hoffmann AA, Langham G (2008) Towards an integrated framework for assessing the vulnerability of species to climate change. PLoS Biol 6(12):2621-2626

You L, Ning X, Liu F, Zhao J, Wang Q, Wu H (2013) The response profiles of HSPA12A and TCTP from Mytilus galloprovincialis to pathogen and cadmium challenge. Fish Shellfish Immunol 35 (2):343-350

Young MD, Wakefield MJ, Smyth GK, Oshlack A (2010) Gene ontology analysis for RNA-seq: accounting for selection bias. Genome Biol 11(2):R14

Zemach A, McDaniel IE, Silva P, Zilberman D (2010) Genome-wide evolutionary analysis of eukaryotic DNA methylation. Science 328(5980):916-919

Zhang G, Fang X, Guo X, Li L, Luo R, Xu F et al. (2012) The oyster genome reveals stress adaptation and complexity of shell formation. Nature 490(7418):49-54

Zhang X, Li Q, Kong L, Yu H (2017a) DNA methylation changes detected by methylation-sensitive amplified polymorphism in the Pacific oyster (Crassostrea gigas) in response to salinity stress. Genes Genomics 39(11):1173-1181

Zhang X, Li Q, Yu H, Kong L (2017b) Effects of air exposure on genomic DNA methylation in the Pacific oyster (Crassostrea gigas). J Fish Sci China 24(4):690-697

Zhu Y, Ren C, Wan X, Zhu Y, Zhu J, Zhou H et al. (2013) Gene expression of Hsp70, Hsp90 and Hsp110 families in normal palate and cleft palate during mouse embryogenesis. Toxicol Ind Health 29(10):915-930 